\title{
Final results of CALDER: kinetic inductance light detectors to search for rare events
}

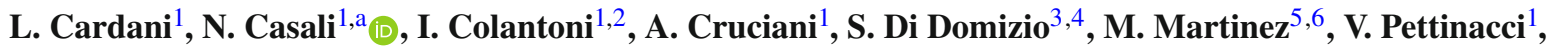 \\ G. Pettinari ${ }^{7}$, M. Vignati $^{1,8}$ \\ ${ }^{1}$ INFN-Sezione di Roma, 00185 Rome, Italy \\ ${ }^{2}$ Consiglio Nazionale delle Ricerche, Istituto di Nanotecnologia (CNR-NANOTEC), c/o Dipartimento di Fisica, Sapienza Università di Roma, \\ 00185 Rome, Italy \\ ${ }^{3}$ Dipartimento di Fisica, Università di Genova, 16146 Genoa, Italy \\ ${ }^{4}$ INFN-Sezione di Genova, 16146 Genoa, Italy \\ ${ }^{5}$ Laboratorio de Física Nuclear y Astropartículas, Universidad de Zaragoza, C/ Pedro Cerbuna 12, 50009 Saragossa, Spain \\ ${ }^{6}$ Fundación ARAID, Av. de Ranillas 1D, 50018 Saragossa, Spain \\ ${ }^{7}$ Consiglio Nazionale delle Ricerche, Istituto di Fotonica e Nanotecnologie (CNR-IFN), Via Cineto Romano 42, 00156 Rome, Italy \\ ${ }^{8}$ Dipartimento di Fisica, Sapienza Università di Roma, 00185 Rome, Italy
}

Received: 14 April 2021 / Accepted: 13 July 2021 / Published online: 21 July 2021

(C) The Author(s) 2021

\begin{abstract}
The next generation of bolometric experiments searching for rave events, in particular for the neutrino-less double beta decay, needs fast, high-sensitivity and easy-toscale cryogenic light detectors. The CALDER project (20142020) developed a new technology for light detection at cryogenic temperature. In this paper we describe the achievements and the final prototype of this project, consisting of a $5 \times 5 \mathrm{~cm}^{2}, 650 \mu \mathrm{m}$ thick silicon substrate coupled to a single kinetic inductance detector made of a three-layer aluminum-titanium-aluminum. The baseline energy resolution is $34 \pm 1$ (stat) \pm 2 (syst) $\mathrm{eV}$ RMS and the response time is $120 \mu \mathrm{s}$. These features, along with the natural multiplexing capability of kinetic inductance detectors, meet the requirements of future large-scale experiments.
\end{abstract}

\section{Introduction}

More than 60 years after their first detection by Cowan and Reines [1], the properties of neutrinos are still being investigated. Among them, the mass scale as well as their nature (Dirac or Majorana particle) are still unknown. If observed, a hypothesized nuclear process could unveil both of them: neutrino-less double beta decay $(0 v \mathrm{DBD})[2,3]$. $0 v$ DBD is not allowed by the Standard Model since it violates the conservation of the B-L number [4]. In this transition a nucleus decays emitting two electrons and no neutrinos, $(\mathrm{A}, \mathrm{Z}) \rightarrow(\mathrm{A}, \mathrm{Z}+2)+2 \mathrm{e}^{-}$, and the signal would consist of

a e-mail: nicola.casali@roma1.infn.it (corresponding author) two electrons with a total kinetic energy equal to the Q-value of the transition. Current upper limits on the half-life of the decay are of the order of $10^{24}-10^{26}$ years, depending on the isotope under study [5-12].

Cryogenic calorimeters, historically also called "bolometers", are among the leading technologies used to search for $0 v \mathrm{DBD}$. The most sensitive experiment based on this technique, CUORE [13], is operating $988 \mathrm{TeO}_{2}$ cryogenic calorimeters of $\sim 750 \mathrm{~g}$ each with an average energy resolution of $7.8 \pm 0.5 \mathrm{keV}$ and a background rate of $(1.49 \pm 0.04) \times$ $10^{-2}$ counts/(keV kg year) in the energy region of interest [14]. These performances allowed the CUORE experiment to set a $90 \%$ C.I. Bayesian lower limit of $2.2 \times 10^{25}$ years on the ${ }^{130} \mathrm{Te}$ half-life for $0 v \mathrm{DBD}$. The CUPID (CUORE Upgrade with Particle Identification) collaboration [15] proposed a next generation $0 \nu \mathrm{DBD}$ experiment to upgrade the technique of CUORE, with the goal of decreasing the background by two orders of magnitude. CUPID will implement an active particle identification technique in order to identify and reject the dominant background source, i.e. $\alpha$ particles emitted by residual radioactive contaminations in the materials facing the detectors [16].

In the first design, CUPID was based on $\mathrm{TeO}_{2}$ cryogenic calorimeters, as in CUORE, complemented by cryogenic light detectors to detect the tiny amount of Cherenkov light $(\sim 100 \mathrm{eV})$ emitted only by $\beta / \gamma$ interactions [17], thus enabling the background rejection. Nevertheless, the results obtained by CUPID-0 [10,18-22] and CUPID-Mo [12,23] experiments demonstrated that the $\alpha$ particles rejection capability can be more easily achieved by using scintillating crys- 
tals like $\mathrm{ZnSe}\left(0 \nu \mathrm{DBD}\right.$ of $\left.{ }^{82} \mathrm{Se}\right)$ and $\mathrm{Li}_{2} \mathrm{MoO}_{4}(0 \nu \mathrm{DBD}$ of ${ }^{100} \mathrm{Mo}$ ) in place of the $\mathrm{TeO}_{2}$ ones because of their higher light signal compared with the Cherenkov yield. Furthermore ${ }^{82} \mathrm{Se}$ and ${ }^{100} \mathrm{Mo}$ benefit from a lower intrinsic background with respect to ${ }^{130} \mathrm{Te}$ because their $0 \nu \mathrm{DBD} \mathrm{Q}$-value is above $2615 \mathrm{keV}$, where the $\beta / \gamma$ background contribution coming from natural radioactivity is greatly reduced. The CUPID group therefore decided to replace $\mathrm{TeO}_{2}$ crystals in favour of $\mathrm{ZnSe}$ or $\mathrm{Li}_{2} \mathrm{MoO}_{4}$. Finally, because of the better radio-purity and energy resolution with respect to $\mathrm{ZnSe}, \mathrm{Li}_{2} \mathrm{MoO}_{4}$ was chosen as crystal for CUPID.

It is clear from the previous considerations that the light detection technology will play a key role in the future of CUPID. To reach the background goal of $10^{-4}$ counts/ (keV kg year), light detectors with small baseline fluctuations $(<100 \mathrm{eV}$ RMS), good time resolution (better than $1 \mathrm{~ms}$ ) and high reproducibility are needed. The light detectors of the first phase of the project will be based on the well established technology of germanium wafers equipped with Neutron Transmutation Doped Germanium (NTD-Ge) thermistors [24]. These sensors offer a typical baseline resolution of 50-60 eV RMS with rise time of 1-5 ms (see, e.g., Refs. $[23,25,26]$ and references therein). A detector of $16 \mathrm{~cm}^{2}$ in an optimized configuration reached an ultimate resolution of $20 \mathrm{eV}$ RMS with rise time of $0.8 \mathrm{~ms}$ [27].

Nevertheless, the physics reach of CUPID (or other bolometric projects such as AMoRE [28]), could be largely increased with faster light detectors. Rise times of the order of hundreds of $\mu \mathrm{s}$ or below would allow to reject the ultimate envisioned background, consisting of pile-up events from the naturally occurring double- $\beta$ decay with the emission of 2 neutrinos [29,30]. Furthermore, detectors offering multiplexing capabilities would allow to increase the number of devices without increasing the thermal load on the cryogenic facility, and ease of fabrication and low-cost readout are highly desirable features for large scale experiments. Apart from the limited time resolution, NTD sensors, being highimpedance devices, cannot be easily multiplexed at cryogenic temperatures.

Light detectors based on transition edge sensors (TES) have also been proposed as an alternative to NTDs for rare events searches [31] and demonstrated an extraordinary energy resolution of $3.9 \mathrm{eV}$ RMS with a distributed network of sensors [32]. Nevertheless the implementation of parallel readout requires further $R \& D$ with respect to the state of the art.

In this work we summarize the $R \& D$ and we present the final results of the CALDER project, which developed an alternative technology to NTDs and TESs for the light detection based on Kinetic Inductance Detectors.
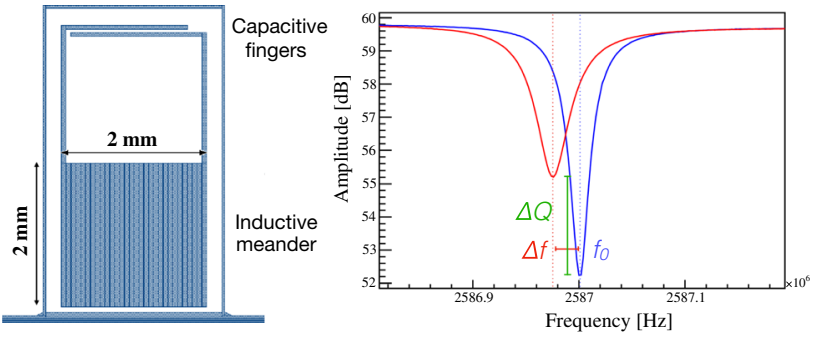

Fig. 1 Left: KID geometry developed in CALDER. The meandered inductor $(L)$ (30 strips of $62.5 \mu \mathrm{m} \times 2 \mathrm{~mm}$ separated by a gap of $5 \mu \mathrm{m})$ has an active area of around $4 \mathrm{~mm}^{2}$, is closed on a 2-finger capacitor $(C)$ on one side, and coupled to a coplanar wave guide (CPW) on the other side. A ground line surrounds the LC circuit for electromagnetic isolation; Right: Frequency scan of a KID. When energy is absorbed in the inductor, the resonant frequency $f_{0}$ and quality factor $Q$ of the resonator are reduced. When the KID is biased on-resonance at fixed readout frequency, the signal is detected as phase and amplitude modulations of the wave transmitted past the device

\section{Kinetic Inductance Detectors with phonon mediation}

In 2003 a new technology was proposed for the detection of electromagnetic radiation at cryogenic temperatures: the Kinetic Inductance Detectors (KIDs) [33]. A KID consist of a resonant LC circuit made of a superconducting metal operated well below its critical temperature $T_{c}$ (see the layout of the KID of CALDER in Fig. 1 left). The inductance $L$ is the sum of the magnetic inductance $\left(L_{g}\right)$, which depends on the geometry of the inductor, and of the so called kinetic inductance $\left(L_{k}\right)$, which depends on the density of Cooper pairs in the superconductor.

For common superconductors such as aluminum, the binding energy of the Cooper pairs is of the order of hundreds of $\mu \mathrm{eV}$, so that even a tiny amount of absorbed energy can break a large number of Cooper pairs. The pair-breaking causes an increase of $L_{k}$ and of the surface resistance of the superconductor, $R$, resulting in a measurable change of the frequency $\left(f_{0}=1 / 2 \pi \sqrt{L C}\right)$ and the quality factor $(1 / Q=R \sqrt{C / L})$ of the resonator (Fig. 1 right).

What makes KIDs interesting with respect to other cryogenic sensors is their natural multiplexing in the frequency domain. Indeed, hundreds of KIDs can be coupled to the same feedline, and can be simultaneously read by making them resonate at slightly different frequencies, which can be obtained by adjusting the layout of the capacitor and/or of the inductor of the circuit [34]. This allowed to successfully operate very large arrays of KIDs, up to 1000 [35]. Despite these outstanding performances, their application in other fields could be limited by their small active surface (few $\mathrm{mm}^{2}$ ). However, Swenson et al. [36] and Moore et al. [37] showed that KIDs are also good phonon sensors: $\mathrm{X}$-rays or, 
more in general, ionising radiation interacting in a substrate generate phonons that, through their scattering in the lattice, can eventually reach the KIDs, break the Cooper pairs and generate a signal. With the mediation of the phonons in the substrate it is therefore possible to increase the sensitive area beyond the KID dimensions, at the cost of a lower sensitivity due to inefficiencies in phonon transmission and absorption in the KID.

\section{The 4 and $25 \mathrm{~cm}^{2}$ light detectors of CALDER}

The CALDER (Cryogenic wide-Area Light Detectors with Excellent Resolution) project [38] developed large area cryogenic light detectors based on KIDs exploiting the phononmediation. The first prototypes $[39,40]$ were made by depositing 4 multiplexed aluminum resonators on a $2 \times 2 \mathrm{~cm}^{2}$, $300 \mu \mathrm{m}$ thick silicon substrate (Fig. 2 left) and reached an energy resolution of $150 \mathrm{eV}$ RMS [41]. Then we improved the layout of the LC circuit in order to increase its area for a better phonon collection (from 2.4 to $4 \mathrm{~mm}^{2}$ ) and in order to increase the quality factor of the resonator (from 15000 to 150000), which in turn needed also an improvement of the quality and accuracy of the metal deposition [42]. Good results were obtained, both in terms of energy resolution and rise time ( $80 \mathrm{eV}$ and $10 \mu \mathrm{s}$ respectively) and also in a wide range of operating temperatures (10-200 mK) [43]. A similar prototype was successfully used as cryogenic light detector coupled to a $2 \times 2 \times 2 \mathrm{~cm}^{3} \mathrm{Li}_{2} \mathrm{MoO}_{4}$, demonstrating very promising performances [44].

In order to further improve the energy resolution, we moved the R\&D to KIDs made of superconductors more sensitive than aluminum. Indeed, the sensitivity depends directly on $L_{k}$ that in turn depends on the material [45]. At the same time the superconducting material needs to feature a good acoustic match to the silicon, to ensure an efficient phonon transmission and absorption in the KID [46]. We started testing several resonators made of sub-stoichiometric titanium nitride without obtaining satisfactory results, mainly because of the poor reproducibility of the film, a difficulty encountered also by other groups $[47,48]$. We then moved to granular aluminum that, because of its tunable energy gap and large fraction of kinetic inductance, is a promising material in applications for quantum circuits [49]. Nevertheless, we were not able to reach a baseline resolution better than $\sim 150 \mathrm{eV}$, likely because of the acoustic mismatch between granular aluminum and silicon, which prevents an efficient collection of phonons. On the contrary, multi-layers of aluminum and titanium showed from the beginning very promising results, thanks to the higher kinetic inductance of titanium. We tested several bi-layers consisting of titanium and aluminum with

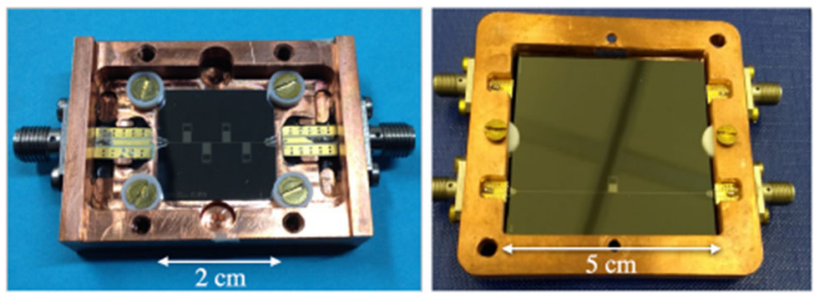

Fig. 2 Left: first prototype of light detector with 4 KIDs coupled to the same feedline and deposited on a $4 \mathrm{~cm}^{2}, 300 \mu \mathrm{m}$ thick, silicon substrate (Picture adapted from Ref. [41]). Right: final prototype with $1 \mathrm{KID}$ deposited on a $25 \mathrm{~cm}^{2}, 650 \mu \mathrm{m}$ thick, substrate

variable thickness. The best baseline resolution, $50 \mathrm{eV}$ RMS, was obtained with $10 \mathrm{~nm}$ of titanium and $25 \mathrm{~nm}$ of aluminum. A further improvement was achieved moving from a bi-layer to a tri-layer of aluminum-titanium-aluminum, in order to enhance the acoustic match between KID and silicon. The best performance was obtained with a three-layer (14 nm Al, $33 \mathrm{~nm} \mathrm{Ti}, 30 \mathrm{~nm} \mathrm{Al}$ ) that, thanks to a 8 times higher kinetic inductance than aluminum, allowed a very competitive baseline energy resolution of $26 \mathrm{eV}$ RMS [50].

The last step of the project consisted in the scale-up of the Si substrate to the final size of $5 \times 5 \mathrm{~cm}^{2}$. Such dimensions were chosen at the beginning of the CALDER R\&D activity to match the size of the faces of the $\mathrm{TeO}_{2}$ crystals of CUORE, in order to maximize the light collection efficiency [17]. Scaling the substrate surface from $2 \times 2$ to $5 \times 5 \mathrm{~cm}^{2}$, and at the same time preserving the baseline resolution, turned out to be the most complex technological challenge. Even if we used the same KID design tested in previous detectors, we had to build and test tens of different prototypes before obtaining a comparable quality factor of the resonator. The reason behind this problem turned out to be the CPW feed line. Indeed, in order to decrease the number of phonons absorbed by non sensitive $\mathrm{Al}$ regions its width was only $85 \mu \mathrm{m}$. At the same time, in order to collect as many phonons as possible, we increased the number of KIDs from 1 to 4, with the feedline running through the whole substrate and being 6 times longer than in the $2 \times 2 \mathrm{~cm}^{2}$ detector. This caused impedance mismatches that reduced by more than an order of magnitude the quality factor. This effect was not visible in EM simulations and therefore we proceeded by trial and error. In the end the simplest solution turned out also to be the most reliable and high-performing: we minimized the feedline length by making it straight from one side of the wafer to the opposite one $(5 \mathrm{~cm})$, and we coupled to it a single KID. Figure 2 (right) shows the final detector held in a copper frame by PTFE washers consisting in a $5 \times 5 \mathrm{~cm}^{2}, 650 \mu \mathrm{m}$ thick Si wafer monitored by an AlTiAl (14 nm, $33 \mathrm{~nm}, 30 \mathrm{~nm}$ ) three-layer resonator with the same geometry showed in Fig. 1 left. 


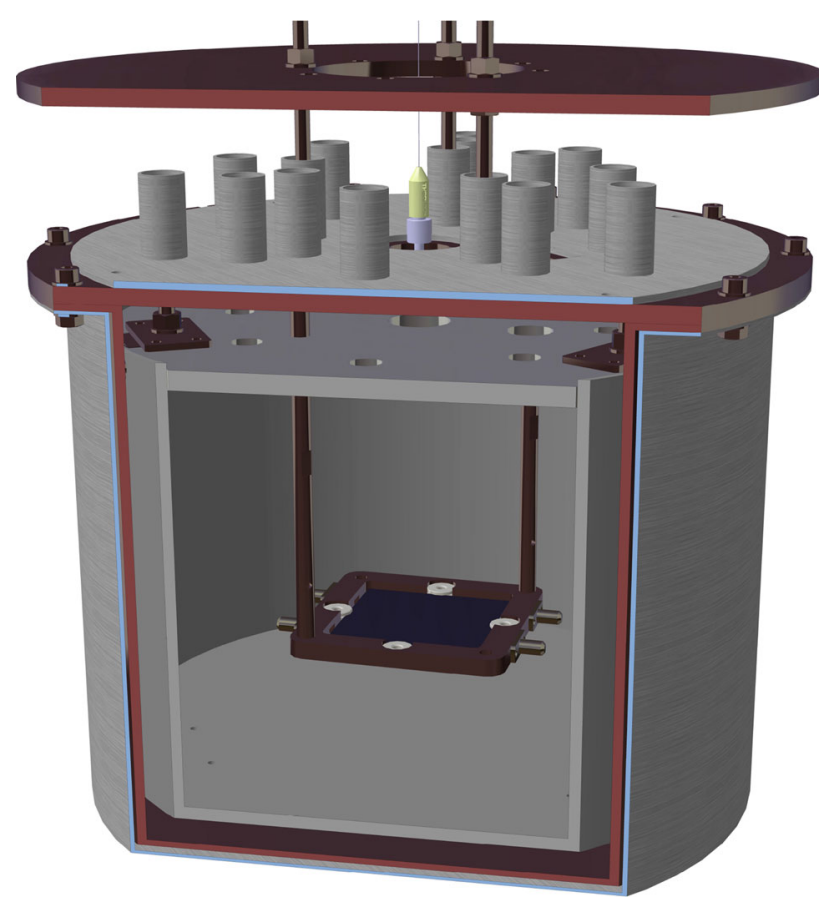

Fig. 3 Rendering of the shielding pot anchored to the $20 \mathrm{mK}$ plate of the cryostat at the top. The pot consists of three concentric layers acting as magnetic and radiation shields. The outer one is made of Cryophy® for magnetic shielding, the middle one of copper for thermalization, and the inner one of aluminum for further magnetic and radiation shielding. The mechanical design of the parts constituting the assembly has been carried out considering the effect of the differential thermal contractions related to the different materials constituting the system (copper, aluminum and Cryophy $\left.{ }^{\circledR}\right)$. In this way, at cryogenic temperatures, the system has maintained minimum gaps without introducing unwanted mechanical stress on the components. The "chimneys" are feedthroughs for cables made of Cryophy®as well. The optical fiber, shown in yellow, illuminates the detector from the top and is connected on the other end to a room temperature LED (not shown in the figure). The detector frame is anchored to the copper shield with two copper bars for thermalization

\section{Measurement set-up}

The device was anchored to the coldest point of a dry ${ }^{3} \mathrm{He} /{ }^{4} \mathrm{He}$ dilution refrigerator ${ }^{1}$ and cooled down to $20 \mathrm{mK}$. The outside vessel of the refrigerator was surrounded by a $\mu$ metal cylinder shield in order to reduced the magnetic field at the sample location. Finally, to prevent that both the residual magnetic field inside the cryostat and the thermal radiation from the $600 \mathrm{mK}$ stage spoiled the resonator quality factor, the detector holder was placed inside a shielding pot consisting in a three layers of Cryophy ${ }^{\circledR}$, copper and aluminum (Fig. 3).

An optical fiber of $600 \mu \mathrm{m}$ core diameter was placed on the top of the pot and illuminated the Si substrate from a distance of $13.6 \mathrm{~cm}$. The opposite end of the fiber was connected

$\overline{1}$ Oxford Instruments, Dry Dilution Refrigerator Triton 200.

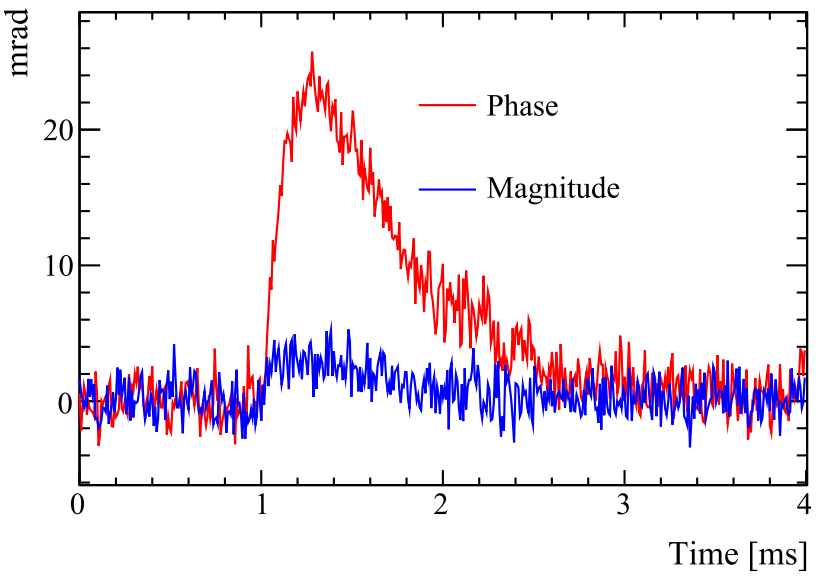

Fig. 4 Triggered $1.3 \mathrm{keV}$ signal in the phase (red) and amplitude (blue) directions

to a $400 \mathrm{~nm}$ LED lamp located at room temperature outside the refrigerator. Given the numerical aperture of the fiber of 0.22 and the distance from the Si surface, photons illuminate the entire $5 \times 5 \mathrm{~cm}^{2}$ surface, thus simulating the crystal scintillation light. A non collimated ${ }^{55} \mathrm{Fe} \mathrm{X}$-ray source was placed at a corner of the Si substrate, on the opposite position with the respect to the KID and the feedline.

The KID resonated at $f_{0}=2.333 \mathrm{GHz}$ and featured a quality factor of 83000 , due to the combination of coupling and internal quality factors amounting to 95000 and 655000 , respectively. The device was excited at the resonant frequency with an input signal of $-75 \mathrm{dBm}$ power and the output was fed into a CITLF3 SiGe cryogenic lownoise amplifier [51] installed at the $4 \mathrm{~K}$ stage of the cryostat, down-converted at room temperature using a superheterodyne electronics and then digitized at a sampling frequency of $500 \mathrm{kSPS}$ [52]. Time traces up to $12 \mathrm{~ms}$ long of the real (I) and imaginary (Q) parts of the transmission $\mathrm{S}_{21}$ were acquired with a software trigger. Finally the I and Q waveforms were converted into phase and amplitude variations relative to the center of the resonance loop [50]. Figure 4 shows a signal produced by an energy deposition of $1.3 \mathrm{keV}$ in the Si wafer, which is similar to the light signal expected from the $0 \nu \mathrm{DBD}$ of ${ }^{100} \mathrm{Mo}$. The average rise time of the signals is $120 \mu \mathrm{s}$, dominated by the phonon propagation in the substrate, while the decay time is $550 \mu \mathrm{s}$, dominated by the recombination of the Cooper pairs [53]. For the estimation of the signal amplitude, the phase and amplitude waveforms are combined with a bi-dimensional matched filter to maximize the signal to noise ratio [43].

\section{Results}

We used the LED lamp to shine different amounts of photons and to perform an absolute energy calibration of the detec- 

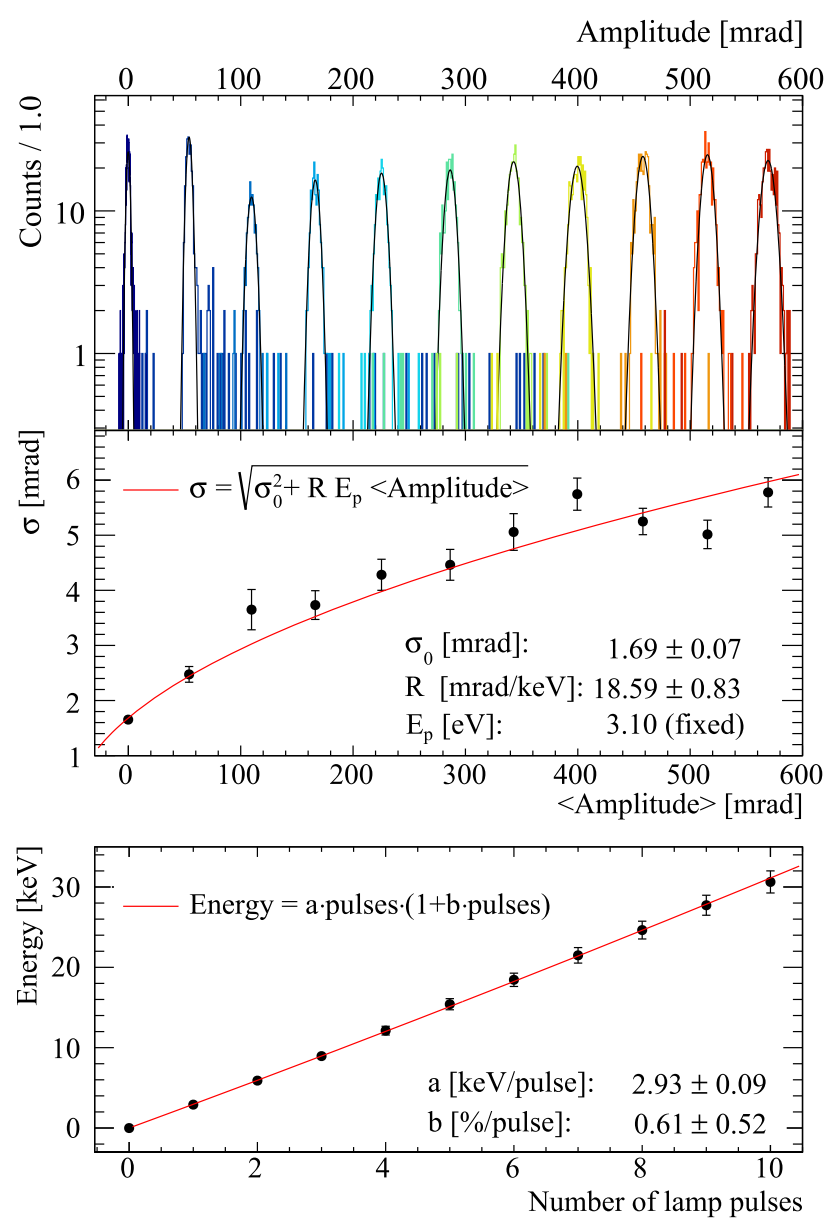

Fig. 5 Absolute energy calibration using a controlled number of fast LED pulses in order to shine increasing amounts of photons in the substrate. Top: Distribution of the amplitude of the signals after the bidimensional matched filter and Gaussian fits to the distributions. Middle: $\sigma$ of the Gaussians as a function of their mean value ( $<$ Amplitude $>$ ) and result of the fit to Eq. (1). Bottom: Calibrated energy as a function of the number of lamp triggers. The fit function shown in the figure is used to estimate the non-linearity $b$ of the calibration procedure and it results negligible

tor [50]. The lamp is controlled with an external trigger that we fire at increasing rate in order to increase the number of photons in a burst. The photon bursts are much shorter than the rise time of the signal, at maximum $2 \mu$ s long, and are therefore seen as a $\delta(t)$ excitation by the KID. Each burst is composed by $\mathrm{N}$ photons of same energy $(400 \mathrm{~nm}$, corresponding to $3.1 \mathrm{eV}$ ) reaching the $\mathrm{Si}$ wafer and absorbed by it. We denote the average number of absorbed photons by $\langle\mathrm{N}\rangle$. This process follows the Poisson statistics and therefore the standard deviation is $\sqrt{<\mathrm{N}\rangle}$.

As shown in Fig. 5 (top) for each optical burst, the distribution of the amplitude of the signals is well described by a Gaussian with a standard deviation $\sigma$. The predicted trend of $\sigma$ as a function of the mean Amplitude ( $<$ Amplitude $>$ ) can be written as the combination of two uncorrelated terms that can be added quadratically, i.e. the poissonian component and the baseline noise energy resolution:

$\sigma=\sqrt{\sigma_{0}^{2}+\mathrm{R} \cdot \mathrm{E}_{\mathrm{p}} \cdot<\text { Amplitude }>}$

where $\sigma_{0}$ is the baseline noise energy resolution, R the energy calibration coefficient and $\mathrm{E}_{\mathrm{p}}$ the photon energy. The $\sigma$ vs $<$ Amplitude $>$ trend is well described by Eq. (1) as shown in Fig. 5-middle and allowed us to extract the calibration coefficient $R=18.59 \pm 0.83 \mathrm{mrad} / \mathrm{keV}$. Finally, the bottom panel of the figure shows the calibrated energy as a function of the number of lamp triggers.

After calibration the baseline noise energy resolution is $90 \mathrm{eV}$, a value obtained with the pulse-tube cryocooler on and without any decoupling system to reduce vibrations (see e.g. Refs. [54,55]). These represent the least favorable configuration, since it is well known that the mechanical vibrations induced by dry refrigerators increase the noise of cryogenic detectors. NTDs and TES detectors, at least in the applications for rare events searches, cannot be even operated without a decoupling system. Indeed, the CUORE infrastructure implements a sophisticated system for the reduction of vibrations [56,57] and to emulate such "ideal" conditions we repeated the measurement with the pulse-tube off and obtained a baseline noise of $34 \pm 1$ (stat) \pm 2 (syst) eV (Fig. 6 top-left), where the systematic error arises from the calibration function.

Figure 6 (bottom) shows the comparison of the noise power spectra of the phase and magnitude readouts with the pulse tube on and off. When the pulse tube is off, the magnitude noise is dominated by the white noise added by the cryogenic amplifier while the phase noise is attributed to two-level systems originating at the interface between the superconductor and the substrate, as observed in our previous devices (see e.g. Ref. [43]). When the pulse tube is on, the increase of the noise at low frequency is evident and does not show evident features in the signal bandwidth, which extends below $500 \mathrm{~Hz}$.

Figure 6 (right) shows the light-calibrated energy spectrum (black histogram) of the events produced by the ${ }^{55} \mathrm{Fe} \mathrm{X}$ ray source. The reconstructed energy is significantly smaller the nominal one $(\sim 6 \mathrm{keV})$, since these devices are sensitive to the position of the energy release [53] and the source, unlike the fiber that illuminates uniformly the substrate, faces one corner of it far from the KID.

\section{Conclusion}

In this paper we described the technological challenges and solutions in the development of wide-area cryogenic light detectors based on Kinetic Inductance Detectors. We presented the final prototype of the CALDER project, a sin- 

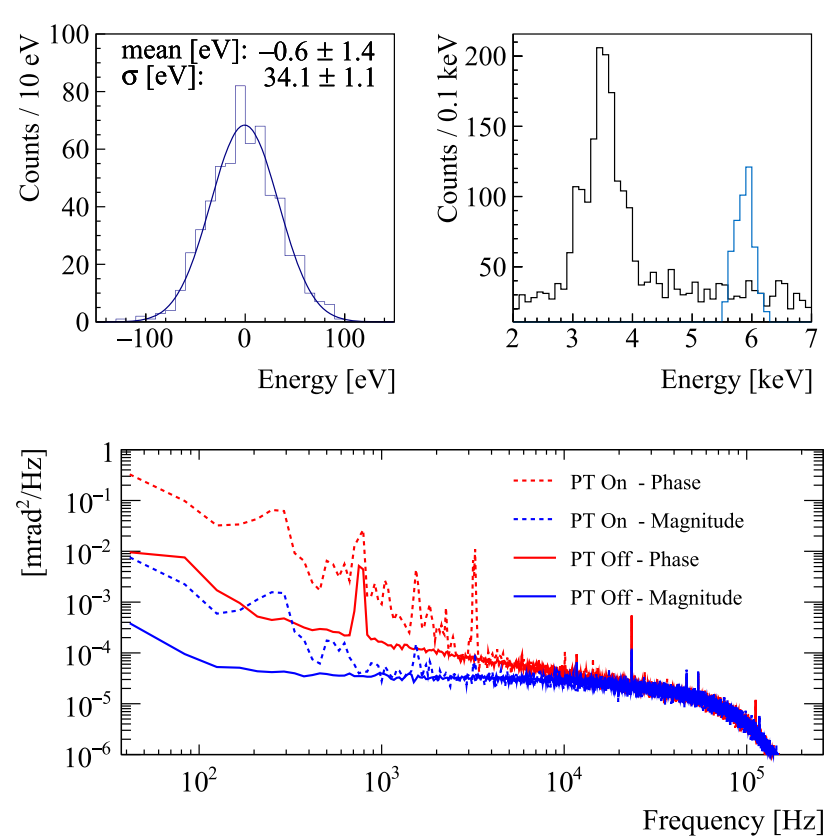

Fig. 6 Left: Light-calibrated energy distribution of noise triggers with the pulse-tube cryocooler off. Bottom: Comparison of the noise power spectra of the magnitude and phase readout with the pulse-tube cryocooler on and off. Right: light-calibrated energy spectrum acquired with the ${ }^{55} \mathrm{Fe}$ source (black) and of optical pulses close to the nominal energy of the source (cyan). The peak of ${ }^{55} \mathrm{Fe}$ is shifted to lower energies because of the position of the source, which fires at a corner of the substrate opposite to the KID position, while the light illuminate the substrate uniformly

gle KID made of a tri-layer aluminum-titanium-aluminum deposited on a $5 \times 5 \mathrm{~cm}^{2}$ Silicon substrate acting as light absorber.

This light detector features a rise time of $120 \mu \mathrm{s}$ and a vibration-limited noise resolution of $90 \mathrm{eV}$ RMS, matching the requirements of next-generation experiments. We proved that the energy resolution is not limited by the detector itself, rather by the vibrations induced by the pulse-tube cryocooler. In absence of such vibrations the energy resolution improves to $34 \mathrm{eV}$ RMS.

Compared to the baseline technology of CUPID, the presented device has a similar energy resolution and an order of magnitude faster response time, a key parameter for background suppression. Finally, it offers ease in multiplexing and simple fabrication, important aspects for experiments with thousands of channels.

Acknowledgements This work was supported by the European Research Council (FP7/2007-2013) under Contract No. CALDER No. 335359 and by the Italian Ministry of Research under the FIRB Contract No. RBFR1269SL. The authors thank M. Calvo, M.G. Castellano, C. Cosmelli, A. Monfardini, H. Le Sueur and I.M. Pop for the precious collaboration and the useful discussions during the development of the project. The authors also thanks the personnel of INFN Sezione di Roma for the technical support, in particular M. Iannone, A. Girardi and S. Casani.
Data Availability Statement This manuscript has no associated data or the data will not be deposited. [Authors' comment: Data will be made available upon reasonable request.]

Open Access This article is licensed under a Creative Commons Attribution 4.0 International License, which permits use, sharing, adaptation, distribution and reproduction in any medium or format, as long as you give appropriate credit to the original author(s) and the source, provide a link to the Creative Commons licence, and indicate if changes were made. The images or other third party material in this article are included in the article's Creative Commons licence, unless indicated otherwise in a credit line to the material. If material is not included in the article's Creative Commons licence and your intended use is not permitted by statutory regulation or exceeds the permitted use, you will need to obtain permission directly from the copyright holder. To view a copy of this licence, visit http://creativecomm ons.org/licenses/by/4.0/.

Funded by $\mathrm{SCOAP}^{3}$.

\section{References}

1. C. Cowan, F. Reines, F. Harrison, H. Kruse, A. McGuire, Science 124, 103-104 (1956). https://doi.org/10.1126/science.124. 3212.103

2. W.H. Furry, Phys. Rev. Lett. 56, 1184 (1936)

3. F. Deppisch, M. Hirsch, H. Päs, J. Phys. G 39, 124007 (2012). https://doi.org/10.1088/0954-3899/39/12/124007

4. F. Vissani et al., Adv. High Energy Phys. 2016, 2162659 (2016). https://doi.org/10.1155/2016/2162659

5. The GERDA Collaboration, Phys. Rev. Lett. 125, 252502 (2020). https://doi.org/10.1103/PhysRevLett.125.252502

6. The KamLAND-Zen Collaboration, Phys. Rev. Lett. 117, 082503 (2016). https://doi.org/10.1103/PhysRevLett.117.082503

7. The Majorana Collaboration, Phys. Rev. C. 100, 025501 (2019). https://doi.org/10.1103/PhysRevC.100.025501

8. The EXO-200 Collaboration, Phys. Rev. Lett. 123, 161802 (2019). https://doi.org/10.1103/PhysRevLett.123.161802

9. The NEMO-3 Collaboration, Phys. Rev. D, 92, 072011 (2015). https://doi.org/10.1103/PhysRevD.92.072011

10. The CUPID-0 Collaboration, Phys. Rev. Lett. 123, 032501 (2019). https://doi.org/10.1103/PhysRevLett.123.032501

11. D.Q. Adams et al. [CUORE Collaboration], Phys. Rev. Lett. 124(12), 122501 (2020). https://doi.org/10.1103/PhysRevLett. 124.122501

12. E. Armengaud et al. [CUPID], Phys. Rev. Lett. 126(18), 181802 (2021). https://doi.org/10.1103/PhysRevLett.126.181802

13. D.R. Artusa et al., Adv. High Energy Phys. 2015, 879871 (2015). https://doi.org/10.1155/2015/879871

14. D.Q. Adams et al. [CUORE], High sensitivity neutrinoless double-beta decay search with one tonne-year of CUORE data. arXiv:2104.06906 [nucl-ex]

15. W.R. Armstrong et al. [CUPID], CUPID pre-CDR. arXiv:1907.09376 [physics.ins-det]

16. C. Alduino et al. [CUORE Collaboration], Eur. Phys. J. C 77(8), 543 (2017). https://doi.org/10.1140/epjc/s10052-017-5080-6

17. N. Casali, Astropart. Phys. 91, 44 (2017). https://doi.org/10.1016/ j.astropartphys.2017.03.004

18. O. Azzolini et al., [CUPID Collaboration], Eur. Phys. J. C 78, 428 (2018). https://doi.org/10.1140/epjc/s10052-018-5896-8

19. The CUPID-0 Collaboration, Phys. Rev. Lett. 123, 262501 (2019). https://doi.org/10.1103/PhysRevLett.123.262501

20. The CUPID-0 Collaboration, Eur. Phys. J. C 78, 888 (2018). https:// doi.org/10.1140/epjc/s10052-018-6340-9 
21. The CUPID-0 Collaboration, Eur. Phys. J. C 80, 702 (2020). https:// doi.org/10.1140/epjc/s10052-020-8280-4

22. The CUPID-0 Collaboration, Phys. Rev. D 100, 092002 (2019). https://doi.org/10.1103/PhysRevD.100.092002

23. E. Armengaud et al., Eur. Phys. J. C 80(1), 44 (2020). https://doi. org/10.1140/epjc/s10052-019-7578-6

24. J.W. Beeman et al., JINST 8, P07021 (2013). https://doi.org/10. 1088/1748-0221/8/07/P07021

25. R.D. Artusa et al., Eur. Phys. J. C 76, 364 (2016). https://doi.org/ 10.1140/epjc/s10052-016-4223-5

26. The CUPID Collaboration, Eur. Phys. J. C 81(2), 104 (2021). https://doi.org/10.1140/epjc/s10052-020-08809-8

27. M. Barucci et al., Nucl. Instrum. Meth. A 935, 150 (2019). https:// doi.org/10.1016/j.nima.2019.05.019

28. V. Alenkov et al., arxiv:1903.09483

29. A. Armatol et al. [CUPID Collaboration], arXiv:2011.11726 [physics.ins-det]

30. D.M. Chernyak et al., Eur. Phys. J. C 77, 3 (2017). https://doi.org/ 10.1140/epjc/s10052-016-4565-Z

31. K. Schäffner et al., Astropart. Phys. 69, 30-36 (2015). https://doi. org/10.1016/j.astropartphys.2015.03.008

32. C.W. Fink et al., Appl. Phys. Lett. 118, 022601 (2021). https://doi. org/10.1063/5.0032372

33. P.K. Day et al., Nature 425, 817-821 (2013). https://doi.org/10. 1038/nature02037

34. B.A. Mazin et al., Proc. SPIE Int. Soc. Opt. Eng. 7735, 773518 (2010). https://doi.org/10.1117/12.856440

35. R. Adam et al., Astron. Astrophys. 609, A115 (2018). https://doi. org/10.1051/0004-6361/201731503

36. L.J. Swenson et al., J. Appl. Phys. 96, 263511 (2010). https://doi. org/10.1063/1.3459142

37. D.C. Moore et al., J. Appl. Phys. 100, 232601 (2012). https://doi. org/10.1063/1.4726279

38. E. Battistelli, et al., Eur. Phys. J. C 75(8), 353 (2015). https://doi. org/10.1140/epjc/s10052-015-3575-6

39. I. Colantoni et al., Nucl. Instrum. Meth. Phys. Res. Sect. A 824, 177-178 (2016). https://doi.org/10.1016/j.nima.2015.10.093
40. I. Colantoni et al., J. Low Temp. Phys. 184, 131-136 (2016). https:// doi.org/10.1007/s10909-015-1452-1

41. L. Cardani et al., Appl. Phys. Lett. 107, 093508 (2015). https://doi. org/10.1063/1.4929977

42. I. Colantoni et al., J. Low Temp. Phys. 193, 726-731 (2018). https:// doi.org/10.1007/s10909-018-1905-4

43. L. Cardani et al., Appl. Phys. Lett. 110(3), 033504 (2017). https:// doi.org/10.1063/1.4974082

44. N. Casali et al., Eur. Phys. J. C 79(8), 724 (2019). https://doi.org/ 10.1140/epjc/s10052-019-7242-1

45. J. Zmuidzinas, Annu. Rev. Cond. Matter Phys. 3, 169 (2012). https://doi.org/10.1146/annurev-conmatphys-020911-125022

46. S.B. Kaplan, J. Low Temp. Phys. 37, 343 (1979). https://doi.org/ 10.1007/BF00119193

47. H.G. Leduc et al., Appl. Phys. Lett. 97, 102509 (2010). https://doi. org/10.1063/1.3480420

48. P. Szypryt et al., Opt. Express 25, 25894-25909 (2017). https:// doi.org/10.1364/OE.25.025894

49. L. Grünhaupt et al., Nat. Mater. 18, 816-819 (2019). https://doi. org/10.1038/s41563-019-0350-3

50. L. Cardani et al., Supercond. Sci. Technol. 31, 075002 (2018). https://doi.org/10.1088/1361-6668/aac1d4

51. http://radiometer.caltech.edu/datasheets/amplifiers/CITLF4.pdf

52. O. Bourrion et al., JINST 6, P06012 (2011). https://doi.org/10. 1088/1748-0221/6/06/P06012

53. M. Martinez et al., Phys. Rev. Appl. 11, 064025 (2019). https://doi. org/10.1103/PhysRevApplied.11.064025

54. S. Pirro, Nucl. Instrum. Meth. A 559, 672-674 (2006). https://doi. org/10.1016/j.nima.2005.12.197

55. R. Maisonobe, J. Billard, M. De Jesus, A. Juillard, D. Misiak, E. Olivieri, S. Sayah, L. Vagneron, JINST 13(08), T08009 (2018). https://doi.org/10.1088/1748-0221/13/08/T08009

56. A. D'Addabbo et al., Cryogenics 93, 56 (2018). https://doi.org/10. 1016/j.cryogenics.2018.05.001

57. C. Alduino et al., Cryogenics 102, 9 (2019). https://doi.org/10. 1016/j.cryogenics.2019.06.011 